\title{
Mini commentary on BJOG-20-1962.R1 'Slower response to treatment of iron deficiency anemia in HIV-infected pregnant women: a prospective cohort study' Anaemia in pregnancy remains a global health problem
}

\author{
Nynke van den Broek ${ }^{1}$ \\ ${ }^{1}$ Centre for Maternal and Newborn Health
}

April 15, 2021

\section{Anaemia in pregnancy remains a global health problem}

In this issue of BJOG Hull et al ..... et al report on an important study from South Africa regarding anaemia in pregnancy and the response to iron therapy. They report that in HIV-positive women the response was slower than in HIV-negative women. The underlying causes of anaemia varied and included iron deficiency (as assessed by ferritin levels) as well as concurrent infections (urinary tract infections and tuberculosis)

Anaemia in pregnancy (blood haemoglobin $\mathrm{Hb}<11.0 \mathrm{~g} / \mathrm{dl}$ ) occurs in $>40 \%$ of women living in low- and middle-income countries (LMIC) and in some settings in Asia prevalence is $>60 \%$. (McCauley et al, BMJ Global Health, 2018; 3(3):e000625) The latest WHO recommendations on antenatal care consider anaemia as the world's second leading cause of disability and one of the most serious global public health problems (WHO Geneva 2016 ).

Although globally the focus has largely been on anaemia in pregnancy resulting from either iron deficiency or malaria, this is an incomplete approach at best. Iron deficiency is hard to measure and confirm as; i) this requires a functioning laboratory to be in place, ii) indicators for iron deficiency are influenced by the presence of concurrent infection, or, iii) repeated measures of $\mathrm{Hb}$ are needed to check whether the anaemia is responsive to treatment with iron. By contrast, malaria is relatively easy to diagnose via rapid diagnostic tests or microscopic examination of a stained blood smear slide. The handful of studies which have comprehensively assessed aetiology of anaemia in pregnant women demonstrate that anaemia is most commonly the result of complex multiple underlying factors including nutritional deficiencies as well as infectious diseases. Both nutritional deficiency and other infections (malaria, tuberculosis) are more likely with HIV-infection which itself can lead to anaemia probably through direct suppression of erythropoiesis.

Hull et al show what was possible in a real-life clinical practice setting. This example of integration of research into clinical practice is laudable and is illustrative of how such integration could result in better services being made available for women in LMIC where burden of disease is high, but diagnostic tests are largely unavailable. It is sobering to realise that the majority of women world-wide will still only be screened for anaemia during pregnancy using 'conjunctival inspection' which is highly inaccurate. (van den Broek et al. Bull WHO 1999; 77(1):15-21) Rapid diagnostic tests are available for Hb, malaria, syphilis, HIV and, more recently, for tuberculosis. We are doing women a dis-service if we cannot offer at least these basic diagnostic tests as part of antenatal care.

To prevent anaemia during pregnancy, the 'fall-back' position is to offer all women daily iron prophylaxis 
(30-60mg elemental iron) - with luck tablets are available that include folic acid (0.4mg) - along with presumptive treatment of malaria (various regimes) in endemic areas. Multi-micronutrients (including the required amount of iron and folic acid) might actually be better but cannot be recommended because of lack of evidence and they are still three times as expensive as iron and folic acid supplementation alone ( $3 \$$ vs $1 \$$ approximately).

WHO recently recommended a better understanding of the aetiology of anaemia. A search on PubMed shows a clear lack of papers on the topic and more good research is needed. Investment in the antenatal care package offered to women is also much needed if we are aiming for a global 'Health for All'. 